\title{
Interstitial pneumonia associated with neoadjuvant chemotherapy in breast cancer
}

\author{
HIDEMI KAWAJIRI, TSUTOMU TAKASHIMA, NAOYOSHI ONODA, \\ SHINICHIRO KASHIWAGI, TETSUROU ISHIKAWA and KOSEI HIRAKAWA
}

Department of Surgical Oncology, Osaka City University Graduate School of Medicine, Osaka 545-8585, Japan

Received September 25, 2012; Accepted February 19, 2013

DOI: $10.3892 / \mathrm{mco} .2013 .87$

\begin{abstract}
Interstitial pneumonia (IP) is a critical adverse event that may occur during anticancer chemotherapy. Physicians should be aware of the possibility of IP, particularly when dealing with patients on neoadjuvant chemotherapy (NAC) since delays in diagnosis and treatment interfere with the scheduled operation. In our institution, fluorouracil, epirubicin and cyclophosphamide (FEC) followed by weekly paclitaxel is the standard NAC regimen for operable breast cancer cases. In the present study, 95 patients with breast cancer were treated with this regimen, 5 of whom (5.3\%) developed IP during NAC. All 5 cases were diagnosed when anticancer therapy was withdrawn and steroid pulse therapy was initiated. Consequently, the standard operation was immediately performed. In conclusion, physicians should be aware that IP may occur at any point during NAC and that it should be immediately diagnosed and treated to avoid delay of the therapeutic plan.
\end{abstract}

\section{Introduction}

Interstitial pneumonia (IP), although infrequently encountered, is a serious adverse event that may occur during anticancer chemotherapy. Two mechanisms have been proposed to explain how anticancer drugs may injure the lung: anticancer drugs damage the lung either directly through cytotoxic effects or indirectly by activating the immune system of the patient. These two mechanisms are involved in the initiation and progression of IP (1). The onset of IP may differ depending on the drug or pathogenic mechanism. Initial symptoms of IP, including pyrexia, coughing and dyspnea, are usually non-specific and unclear, and sometimes delay the appropriate diagnosis (2). IP is significant since a prolonged illness or severe respiratory sequelae could interfere with subsequent

Correspondence to: Dr Hidemi Kawajiri, Department of Surgical Oncology, Osaka City University Graduate School of Medicine, 1-4-3 Asahi-machi, Abeno-ku, Osaka 545-8585, Japan

E-mail: hkawajiri75@yahoo.co.jp

Key words: interstitial pneumonia, neoadjuvant chemotherapy, breast cancer anticancer treatments. Moreover, IP itself could be fatal when not managed appropriately (2).

Neoadjuvant chemotherapy (NAC) is a promising treatment for operable breast cancer cases since its feasibility and efficacy have been previously demonstrated (3-6). It is now the treatment of choice for patients with breast cancer as it effectively improves the rate of breast conservation, as well as the prognosis of the patient in a practical, clinical setting $(3,4)$. Fluorouracil, epirubicin and cyclophosphamide (FEC) followed by weekly paclitaxel are the standard NAC regimen in patients with operable advanced breast cancer used in our institution. The pathological complete response rate (pCR) of the patients treated with this regimen is as high as $39 \%$. Additionally, this regimen has been administered effectively in the out-patient setting and no life-threatening adverse events have been observed (unpublished data).

The clinical courses of 5 patients who developed IP during NAC at our institution are described in the present study. The importance of an early diagnosis and treatment that promote immediate recovery and prevent critical delays in the subsequent anti-cancer therapy is discussed.

\section{Patients and methods}

Patients with IP during NAC. The adverse events of 95 women with locally advanced breast cancer, who had been treated with FEC followed by weekly paclitaxel between December, 2005 and February, 2010, were retrospectively analyzed. Five patients discontinued NAC due to IP (5.3\%). The background characteristics of these 5 patients are provided in Table I. These patients were female, aged 45-63 years (average, 53). Clinically, their initial diseases ranged from stage IIA to IIIC. One case also had multiple sclerosis (Case 3) and another was a hepatitis B virus carrier (Case 4). These diseases were not aggravated by the therapy.

$N A C$. The FEC 100 regimen involved intravenous administration of $500 \mathrm{mg} / \mathrm{m}^{2}$ fluorouracil, $500 \mathrm{mg} / \mathrm{m}^{2}$ cyclophosphamide and $100 \mathrm{mg} / \mathrm{m}^{2}$ epirubicin on day 1 . This regimen was administered every 21 days for 4 cycles, followed by a once-weekly intravenous infusion of $80 \mathrm{mg} / \mathrm{m}^{2}$ paclitaxel for 12 weeks. Since all the cases had HER-2-negative tumors, an anti-Her2 agent was not administered during NAC. The protocol of this NAC regimen was approved by the Chemotherapeutic Committee of 
Table I. Characteristics of 5 patients with interstitial pneumonia.

\begin{tabular}{lcccccc}
\hline Case & Age (years) & Stage & ER & PgR & HER2 & Associated disease \\
\hline 1 & 45 & IIIA & + & + & - & - \\
2 & 50 & IIIB & - & - & - & - \\
3 & 48 & IIB & - & - & - & Multiple sclerosis \\
4 & 63 & IIA & + & + & HBV carrier \\
5 & 59 & IIIC & + & + & - & - \\
\hline
\end{tabular}

ER, expression of estrogen receptor by the tumor; PgR, expression of progesterone receptor by the tumor; HER2, expression of human epidermal growth factor receptor 2 by the tumor; HBV, hepatitis B virus.
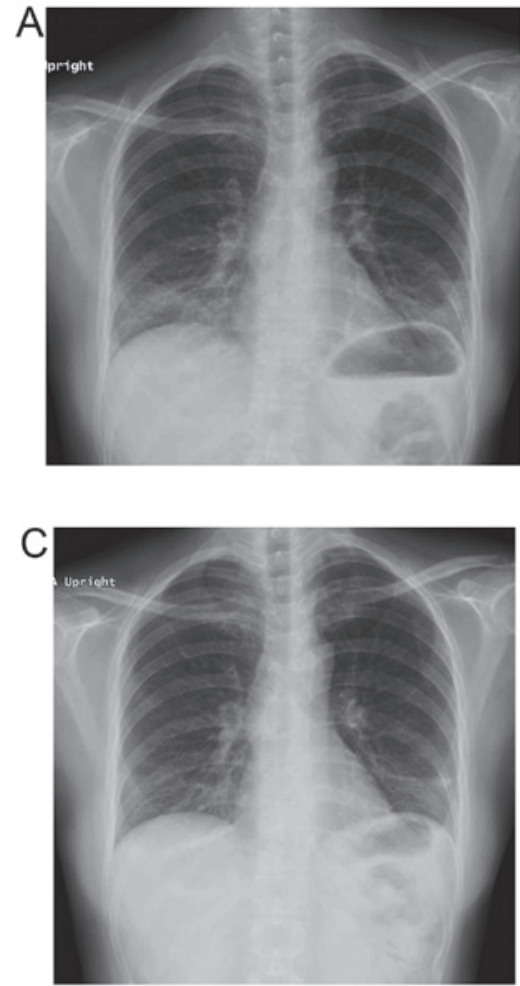

$\mathrm{B}$

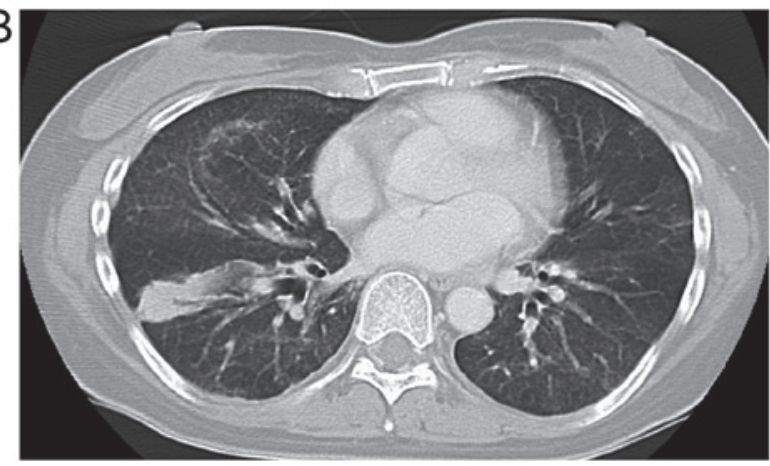

D

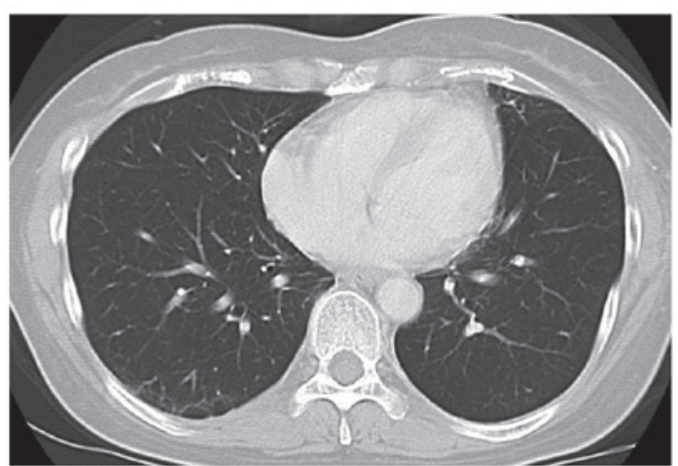

Figure 1. Case 1. (A) Chest X-ray on admission shows diffuse and interstitial infiltrate in the bilateral lower lung fields. (B) Chest computed tomography (CT) on admission shows ground glass opacity in the bilateral lungs, with focal consolidation and mild traction bronchiectasis. (C) Chest X-ray following steroid pulse therapy shows an improvement in the interstitial infiltrate. (D) Chest CT following steroid pulse therapy shows that the interstitial infiltrate has disappeared.

the Osaka City University Hospital (Osaka, Japan) and written informed consent was obtained from each patient.

\section{Results}

Representative case (Case 1). A 45-year-old female was diagnosed with left breast cancer (T2 N1 M0 stage IIB) and NAC regimen was administered. The patient became aware of a cough and general fatigue 4 days following the 10th once-weekly paclitaxel administration. Since an abnormal shadow was not observed on chest X-ray, the 11th scheduled once-weekly paclitaxel was administered. The cough and general fatigue worsened and the patient began to exhibit pyrexia and hypoxia, despite the administration of antibiotics. Chest X-ray indicated ground glass opacity on the bilateral lower lung field (Fig. 1A). A chest CT performed on the same day showed diffuse ground glass opacity in the bilateral lower lung field (Fig. 1B), and IP was diagnosed. NAC (the 12th and last administration of once-weekly paclitaxel) was suspended and steroid pulse therapy (methylprednisolone $1 \mathrm{~g}$ for 3 days) was initiated upon admission. The 12th paclitaxel dose was not administered. The symptoms immediately improved and the abnormal X-ray and CT images were eluminated (Fig. 1C and D). The patient was discharged on the 5th hospital day. Breast-conserving surgery with axillar lymph node dissection was performed 25 days following the last dose of NAC, which was not behind the scheduled timing. Local radiation therapy (50 Gy) was then added as scheduled without complications. Pathological examination showed no residual cancer cells. Goserelin and tamoxifen were administered as adjuvant therapy for 37 months following the operation, and there were no signs 
Table II. Clinical characteristics and course of 5 patients with interstitial pneumonia (IP).

\begin{tabular}{|c|c|c|c|c|c|c|c|c|c|c|c|}
\hline Case & Symptoms & $\begin{array}{l}\text { Onset during } \\
\text { therapy }\end{array}$ & $\begin{array}{c}\mathrm{SpO}_{2} \\
(\%)\end{array}$ & $\begin{array}{l}\text { WBC } \\
(/ \mu 1)\end{array}$ & $\begin{array}{c}\text { CRP } \\
(\mathrm{Mg} / \mathrm{dl})\end{array}$ & $\begin{array}{c}\text { Span from } \\
\text { onset to IP } \\
\text { therapy (days) }\end{array}$ & $\begin{array}{c}\text { Therapy } \\
\text { for IP }\end{array}$ & $\begin{array}{c}\text { Admission } \\
\text { period (days) }\end{array}$ & $\begin{array}{c}\text { Time from } \\
\text { diagnosis to } \\
\text { operation (weeks) }\end{array}$ & $\begin{array}{c}\text { Type of } \\
\text { operation }\end{array}$ & $\begin{array}{l}\text { Effect } \\
\text { of NAC }\end{array}$ \\
\hline 1 & $\mathrm{C}, \mathrm{Fa}, \mathrm{Fe}$ & 11 th & 93 & 5,500 & 1.84 & 10 & MPSL & 5 & 4 & $\mathrm{Bp}+\mathrm{Ax}$ & $\mathrm{pCR}$ \\
\hline 2 & $\mathrm{C}, \mathrm{Fa}, \mathrm{Fe}$ & 9th & 90 & 2,600 & 17.56 & 13 & MPSL $\rightarrow$ PSL & 11 & 5 & $\mathrm{Bt}+\mathrm{Ax}$ & PR \\
\hline 3 & $\mathrm{C}, \mathrm{Fa}$ & $3 \mathrm{rd}$ & 99 & 2,200 & 0.43 & 14 & - & - & 3 & $\mathrm{Bp}+\mathrm{Ax}$ & $\mathrm{pCR}$ \\
\hline 4 & $\mathrm{C}, \mathrm{Fa}, \mathrm{Fe}$ & 8th & 89 & 5,000 & 9.77 & 13 & MPSL $\rightarrow$ PSL & 14 & 6 & $\mathrm{Bp}+\mathrm{Ax}$ & PR \\
\hline 5 & $\mathrm{Fa}, \mathrm{Fe}$ & 6th & 94 & 3,800 & 5.28 & 8 & MPSL (0.5 g) & - & 5 & $\mathrm{Bt}+\mathrm{Ax}$ & PR \\
\hline
\end{tabular}

$\mathrm{C}$, cough; $\mathrm{Fa}$, fatigue; Fe, fever; onset during therapy, the number of weekly paclitaxel treatments that were administered prior to $\mathrm{IP}_{\mathrm{P}}$ onset; $\mathrm{SpO}_{2}$, saturation of peripheral oxygen; WBC, white blood cell; CRP, C-reactive protein; MPSL, methyl-prednisolone [intravenously (i.v.); $1 \mathrm{~g} / \mathrm{day}$ for 3 days]; PSL, prednisolone [per os (p.o.); $0.5 \mathrm{mg} / \mathrm{kg}$ ]; Bp, breast conserving surgery; Bt, total mastectomy; Ax, axillary dissection; NAC, neoadjuvant chemotherapy; pCR, pathological complete response; PR, partial response.

Table III. Efficacy of NAC: comparison of cases with and without interstitial pneumonia (IP).

\begin{tabular}{lcc}
\hline & With IP & Without IP \\
\hline No. & 5 & 90 \\
Average age (years) & 53.0 & 52.4 \\
Stage II/III & $2 / 3$ & $66 / 24$ \\
Response rate & $100 \%$ & $92.2 \%$ \\
pCR rate & $40 \%$ & $37.8 \%$ \\
Total dose of paclitaxel & $592 \mathrm{mg} / \mathrm{m}^{2}$ & $960 \mathrm{mg} / \mathrm{m}^{2}$ \\
\hline
\end{tabular}

pCR, pathological complete response.

of recurrent disease. The patient was also free of any respiratory symptoms.

Clinical course of the 5 patients with IP. The clinical course of IP in the 5 patients is shown in Table II. Most patients developed cough, phlegm, general fatigue and pyrexia. Hypoxia occurred in 3 cases. In all the cases, it was possible to initiate treatment for IP within 2 weeks (8-14 days) from the appearance of the initial symptoms. In one case with mild symptoms (Case 3 ), IP was completely resolved by interrupting the NAC regimen. Steroid pulse therapy was required in 2 cases, and additional treatment with prednisolone for 3 weeks was required in the remaining 2 cases. Three patients had to be admitted to the hospital (3-11 days) due to hypoxia. In all the cases, the curative operation was accomplished 3-6 weeks after the last administration of the anticancer drug. Following recovery from IP, paclitaxel administration was not resumed in any of the cases.

Comparison of the five IP cases with patients without IP. The 5 IP cases were compared with other cases administered NAC during the same period in our institution (Table III). Age, stage of breast cancer, response rate, and pCR rate were not significantly different between the 2 groups. Although the patients with IP were administered lower total doses of paclitaxel compared to those without IP (since NAC was interrupted), the efficacy of the treatment was not significantly reduced.

\section{Discussion}

FEC followed by weekly paclitaxel is used as a standard NAC regimen for patients with operable advanced breast cancer in our institution. Although IP has been reported to occur during FEC and paclitaxel therapy (2,7), IP was diagnosed during the administration of paclitaxel in all the cases reported in this study. However, it was not possible to determine whether FEC or paclitaxel induced IP as it occurred at any time following the initiation of administration. However, a clinical study has shown that the incidence of IP during paclitaxel treatment is $<1 \%$ (8). By contrast, 5 cases with IP are described in the present study $(5.3 \%)$. It is therefore possible that preceding paclitaxel therapy with FEC may have had an additional effect regarding the occurrence of IP, potentially by increasing injury to the lung.

Febrile neutropenia is one of the most common adverse events that may occur during chemotherapy and patients usually recover within a few days when antibiotics and granulocyte-colony stimulating factor are provided. However, as the present study has shown, in cases where abnormal inflammation indices are prolonged, a chest CT should be performed to exclude the possibility of IP. The criteria used for diagnosing drug-induced IP include the following: prior administration of a drug that could induce IP; the presence of clinical observations, imaging and pathological findings that do not contradict the possibility of drug-induced IP; improvement of IP symptoms following withdrawal from administration or steroid therapy; recurrence of symptoms when the drug is administered again (9). In acute-type IP, the main symptoms are pyrexia, dry cough, dyspnea, and sometimes respiratory insufficiency. However, in chronic-type IP, the mild respiratory symptoms gradually progress from several weeks to several months. Additionally, the onset of pneumonia is modified by genetic factors, age, total drug dose, additional cytotoxic drug therapy, prior or concurrent radiotherapy treatment, pre-existing pulmonary disease, history of smoking, oxygen therapy, bone marrow or stem cell transplantation, and renal insufficiency (10). Therefore, it is sometimes difficult to determine a final diagnosis of IP based on clinical manifestations alone. As a result, the diagnosis of drug-induced IP often depends on imaging findings as well as clinical symptoms. 
Although clinical imaging findings vary depending on the pathology pattern, ground glass opacity in chest X-rays or CT is the main characteristic of IP (11). Therefore, in cases with suspected respiratory disease during chemotherapy, it may be necessary to perform imaging investigations to diagnose IP.

Two mechanisms are believed to initiate drug-induced IP following treatment with anticancer drugs: the drugs may injure the lung directly via their cytotoxic effects or indirectly by affecting the immune system (1). However, the two mechanisms may act together to induce IP (1). In the present study, symptoms improved immediately following the termination of cytotoxic drug treatment and the initiation of steroid therapy. Thus, both the drug itself and alterations in the immune system may be involved in the initiation of IP.

The standard drug therapy for IP is $0.5-1.0 \mathrm{mg} / \mathrm{kg}$ of prednisolone/day (12). Steroid pulse therapy (1,000 mg of methyl-prednisolone/day for 3 days) may be necessary for cases with severe symptoms. The 4 cases treated with steroid pulse therapy in this study showed significant improvement in their IP symptoms, suggesting that steroid pulse therapy is suitable for inducing an immediate response in cases of drug-induced IP during NAC.

Since patients administered chemotherapy should be considered as compromised hosts, Pneumocystis pneumonia and cytomegalovirus infection should be excluded. Therefore, in the present study, high spectrum antibiotics, co-trimoxazole, and antiviral medication were provided during the steroid therapy until it was clear that these infectious origins were not present, as indicated by negative results of the tests measuring $\beta$-D-glucan and C7-HRP.

Regarding the management of IP that arises during chemotherapy, the most important objectives are to immediately diagnose drug-induced IP and to suspend chemotherapy in order to prevent disease aggravation. Therefore, physicians should always be aware of the fact that IP may occur at any time during chemotherapy, in particular for patients who are administered NAC. This is because IP may interfere with scheduled future anticancer treatment plans, particularly when it is prolonged or aggravated by delays in appropriate management. Furthermore, it should be emphasized that early steroid pulse therapy allows for a rapid and complete cure of IP that arises during chemotherapy.

\section{References}

1. Delaunois LM: Mechanisms in pulmonary toxicology. Clin Chest Med 25: 1-14, 2004

2. Nagata S, Ueda N, Yoshida Y, et al: Severe interstitial pneumonitis associated with the administration of taxanes. J Infect Chemother 16: 340-304, 2010.

3. Wolmark N, Wang J, Mamounas E, et al: Preoperative chemotherapy in patients with operable breast cancer: nine-year results from National Surgical Adjuvant Breast and Bowel Project B-18. J Natl Cancer Inst Monogr 30: 96-102, 2001.

4. van der Hage JA, van de Velde CJ, Julien JP, et al: Preoperative chemotherapy in primary operable breast cancer: results from the European Organization for Research and Treatment of Cancer trial 10902. J Clin Oncol 19: 4224-4237, 2001.

5. Bear HD, Anderson S, Brown A, et al: The effect on tumor response of adding sequential preoperative docetaxel to preoperative doxorubicin and cyclophosphamide: preliminary results from National Surgical Adjuvant Breast and Bowel Project Protocol B-27. J Clin Oncol 21: 4165-4174, 2003.

6. Bear HD, Anderson S, Smith RE, et al: Sequential preoperative or postoperative docetaxel added to preoperative doxorubicin plus cyclophosphamide for operable breast cancer: National Surgical Adjuvant Breast and Bowel Project Protocol B-27. J Clin Oncol 24: 2019-2027, 2006.

7. Segura A, Yuste A, Cercos A, et al: Pulmonary fibrosis induced by cyclophosphamide. Ann Pharmacother 35: 894-897, 2001.

8. Khan A, McNally D, Tutschka PJ and Bilgrami S: Paclitaxelinduced acute bilateral pneumonitis. Ann Pharmacother 31: 1471-1474, 1997.

9. Ellis SJ, Cleverley JR and Müller NL: Drug-induced lung disease: high-resolution CT findings. AJR Am J Roentgenol 175: 1019-1024, 2000.

10. Camus PH, Foucher P, Bonniaud PH and Ask K: Drug-induced infiltrative lung disease. Eur Respir J Suppl 32: S93-S100, 2001.

11. Elliot TL, Lynch DA, Newell JD Jr, et al: High-resolution computed tomography features of nonspecific interstitial pneumonia and usual interstitial pneumonia. J Comput Assist Tomogr 29: 339-345, 2005.

12. Wang GS, Yang KY and Perng RP: Life-threatening hypersensitivity pneumonitis induced by docetaxel (taxotere). Br J Cancer 85: 1247-1250, 2001. 